\title{
Mesure instantanée et continue de la qualité de l'eau.
}

\section{Instantaneous and continuous measurements of water quality}

\author{
Dominique Grange \\ Patrick Marchandise
}

Alain Paitry

Guy Randon

\author{
Laboratoire régional de l'Ouest Parisien \\ Responsable de la section chimie, pollution, épuration \\ Laboratoire central des Ponts et Chaussées \\ Chef de la section pollution assainissement - Division Eau. \\ Service départemental d'assainissement de la Seine-Saint-Denis \\ Ingénieur à la Compagnie générale des eaux
}

Les indicateurs qui permettent d'apprécier la qualité d'une eau - eau-usée, eau pluviale, eau de rivière - sont nombreux et sont choisis selon l'usage ou de la destination assignés à cette eau. La mesure in situ, continue et instantanée nécessite le choix du ou des (2 ou 3) paramètre (s) caractérisant de la manière la plus satisfaisante la qualité de l'eau. Dans le domaine de l'assainissement les mesures de la matière en suspension ou de la matière organique semblent pouvoir être corréléés avec des mesures optiques (visible, ultra violet) accessibles facilement. Plusieurs appareils sont l'objet d'expérimentations qui seront développées dans la communication. Il existe des approches totalement différentes notamment dans le domaine de la mesure en rivière.

Many indicators may characterize water quality - waste water, urban hunoff water, river water and they depend on the use fixed to this water. A continuous in situ measurement requires to choose two or three parameters which characterize water quality as well as possible. Simple optic measures (visible and UV) seem to be reliable with suspended solid and organic substance measures. This paper deals with the experimentation of several measurement equipments.

Dans le domaine de l'assainissement, les techniciens ont fréquemment besoin de mesures qualitatives. Une mesure instantanée et continue de la qualité des eaux fait apparâ̂tre des perspectives très intéressantes dans les domaines de la gestion des ouvrages et de la connaissance des flux [1] [2] :

- gestion optimisée des réseaux pluviaux ou unitaires par temps sec ou lors des ruissellements afin d'améliorer le fonctionnement actuel basé sur des informations hydrauliques;

- optimisation du fonctionnement des stations d'épuration des collectivités, asservissement à la charge et contrôle de la qualité des eaux traitées [1] [2];

- connaissance de la qualité des effluents industriels, contrôle des rejets, calcul de la redevance d'assainissement.
Des essais ont été réalisés dans le passé en adaptant des appareils de laboratoire ou des appareils de mesure en rivière, sans grands succès. Il convient de remarquer qu'il existe sur le marché peu d'appareils adaptés à ce type de mesure. Les mesures directes d'indicateurs de qualité par des méthodes proches de celles utilisées en laboratoire pour les normes AFNOR à l'aide d'automates (ex : DCO mètre) s'avèrent complexes et leur utilisation en réseaux d'assainissement ou en stations d'épuration de tailles moyennes restent problématiques.

Les recherches se sont orientées vers la mesure optique présentant l'avantage de la simplicité [3] [4] [5] [6] [7]. Les premières mesures optiques datent d'une quinzaine d'années et l'arrivée sur le marché de nouveaux appareils technologiquement plus évolués - fibre optique, mesure dans I'UV et le visible - a suscité un regain d'intérêt des chercheurs. 


\section{Tableau 1}

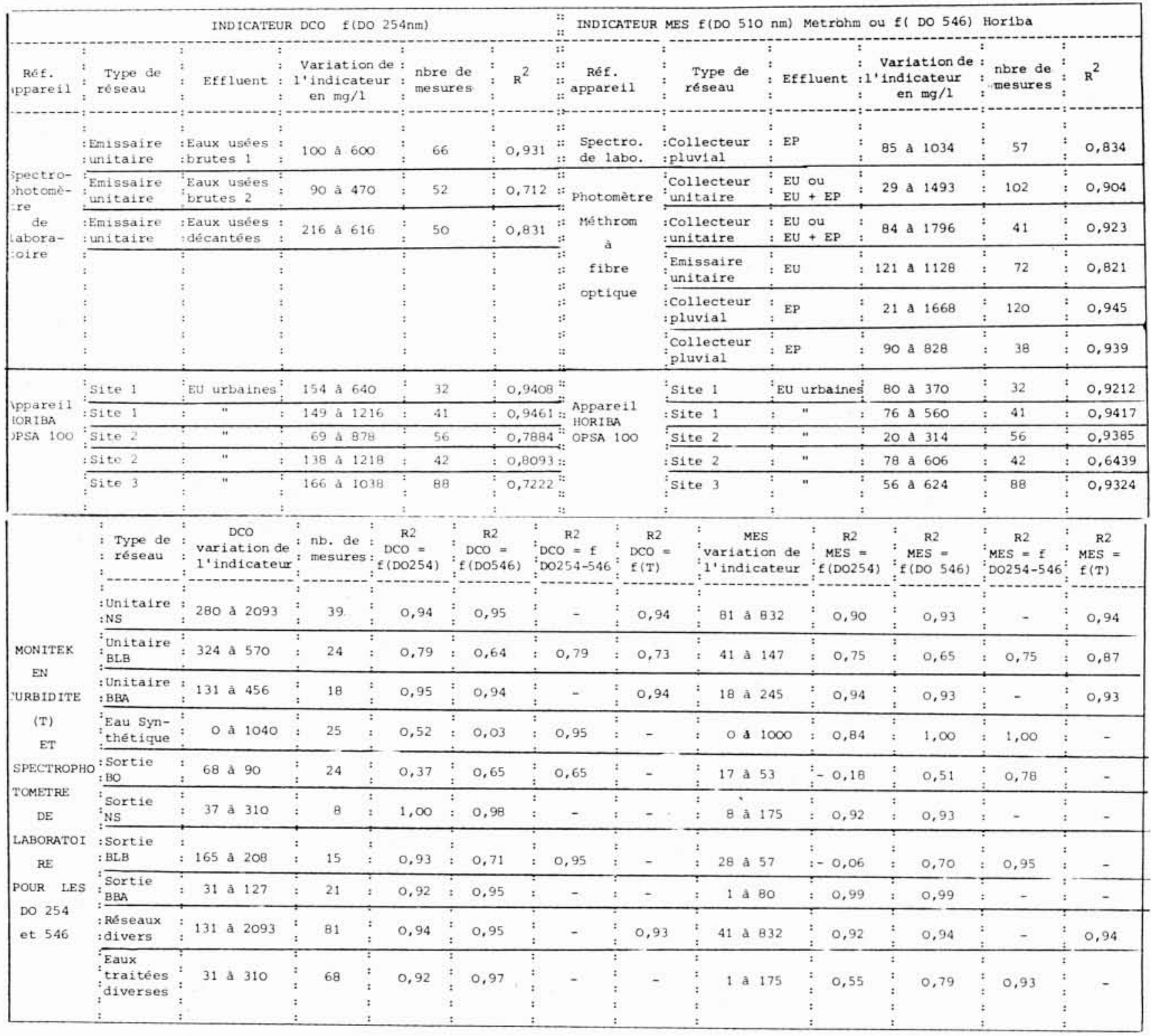

\section{Essais préliminaires}

Des essais en laboratoire utilisant soit des spectrophotomètres classiques, soit les matériels décrits ci-après ont été effectués dans un premier temps afin de vérifier l'existence de relations entre les mesures optiques et les indicateurs de qualité.

Ainsi le laboratoire du Centre de Recherche de la Compagnie Générale des Eaux a réalisé pour la ville de Paris une étude sur les grands émissaires de la région parisienne qui a permis d'établir de bonnes corrélations entre la mesure de la densité optique à 254 nm (DO 254) et les mesures de DCO; (tableau 1).

Le laboratoire régional de l'Est Parisien a réalisé pour le Service départemental d'assainissement de la SeineSaint-Denis de nombreuses expérimentations en labora- toire avec un spectrophotomètre, muni d'un guide optique aboutissant à une cellule ouverte, afin d'établir des relations entre les concentrations en MES et la mesure de la densité optique (DO) ou absorbance. Les mesures ont été réalisées à l'aide d'un appareil Metrohm sur des eaux en provenance de collecteurs unitaires, par temps sec et par temps de pluies, ainsi que sur des collecteurs pluviaux lors de ruissellements. Les corrélations obtenues sont tout à fait satisfaisantes (tableau 1).

Le Laboratoire central des Ponts et Chaussées a recherché d'une part des corrélations simples entre les paramètres de pollution (DCO et MES) et la turbidité (T) déterminée à l'aide de l'appareil Monitek décrit ci-après et l'autre part des corrélations simples et multiples entre ces paramètres de pollution et les densités optiques à $254 \mathrm{~nm}$ et $546 \mathrm{~nm}$ (DO 254 et 546 ). 
Les mesures ont été réalisées sur 7 sites ( 3 en réseaux d'assainissement, 4 sur eaux traitées par une station d'épuration biologique) et sur des échantillons synthétiques (viandox et kaolin représentant respectivement DCO et MES).

Des analyses granulométriques n'ont pas permis de mettre en évidence sur les échantillons examinés une influence de la taille des particules sur les mesures optiques.

Sur échantillons synthétiques dont les DCO et MES variaient indépendamment, nous observons que les MES ont une incidence sur les DO 254 et que par conséquent la DCO ne peut être exprimée valablement que par une combinaison des DO 254 et 546 . Sur réseaux urbains, nous pouvons considérer que DO 546 et DO-254 ou T permettent d'estimer DCO et MES avec une précision suffisante.

Sur eaux traitées, la précision des relations est dépendante de la plage de variation de la pollution des échantillons sur les divers sites mais une utilisation conjointe de la DO-254 et de la DO 546 permet d'avoir une estimation plus précise de la pollution [8].

Des tests ont été menés en laboratoire avec l'appareil Methrom muni d'un guide optique afin de connaitre l'influence de la longueur d'onde de mesure, l'influence des substances colorées minérales et organiques, l'encrassement de la cellule et l'influence de la granulométrie. La réponse est très dépendante de la granulométrie, les autres paramètres ne perturbent que peu ou pas la mesure. Toutefois pour un type d'effluent, les variations granulométriques entre échantillons ne sont pas suffisamment importantes pour perturber la mesure de manière significative.

\section{Description des appareils}

\subsection{L'analyseur Horiba OPSA-100 (fig. 1)}

Cet appareil effectue la mesure simultanée de l'absorption dans l'ultra violet à $254 \mathrm{~nm}$, représentative de la pollution organique, et de l'absorption dans le visible à $546 \mathrm{~nm}$, liée à la turbidité de l'effluent.

Il est constitué :

- d'une cuve à niveau constant alimentée par une pompe extérieure:

- d'une cellule de mesure mise en place dans la cuve (fig. 2);

- d'un boitier électronique pour l'étalonnage de la cellule de mesure et l'exploitation des signaux.

L'appareil peut ainsi calculer automatiquement les corrections dues à la turbidité et fournir un signal de la forme DO 254-DO 546.

Le dispositif de détection, immergé dans le bac, est constitué d'une cellule émettrice et d'une cellule réceptrice en rotation excentrée.

Outre l'auto-nettoyage des cellules par des balais, ce mouvement permet de faire varier la longueur du trajet optique pour fournir une mesure différentielle qui ne dépend que de l'absorption propre à l'échantillon. L'appareil s'affranchit ainsi de toutes les sources de dérive liées en particulier à l'encrassement des cellules.
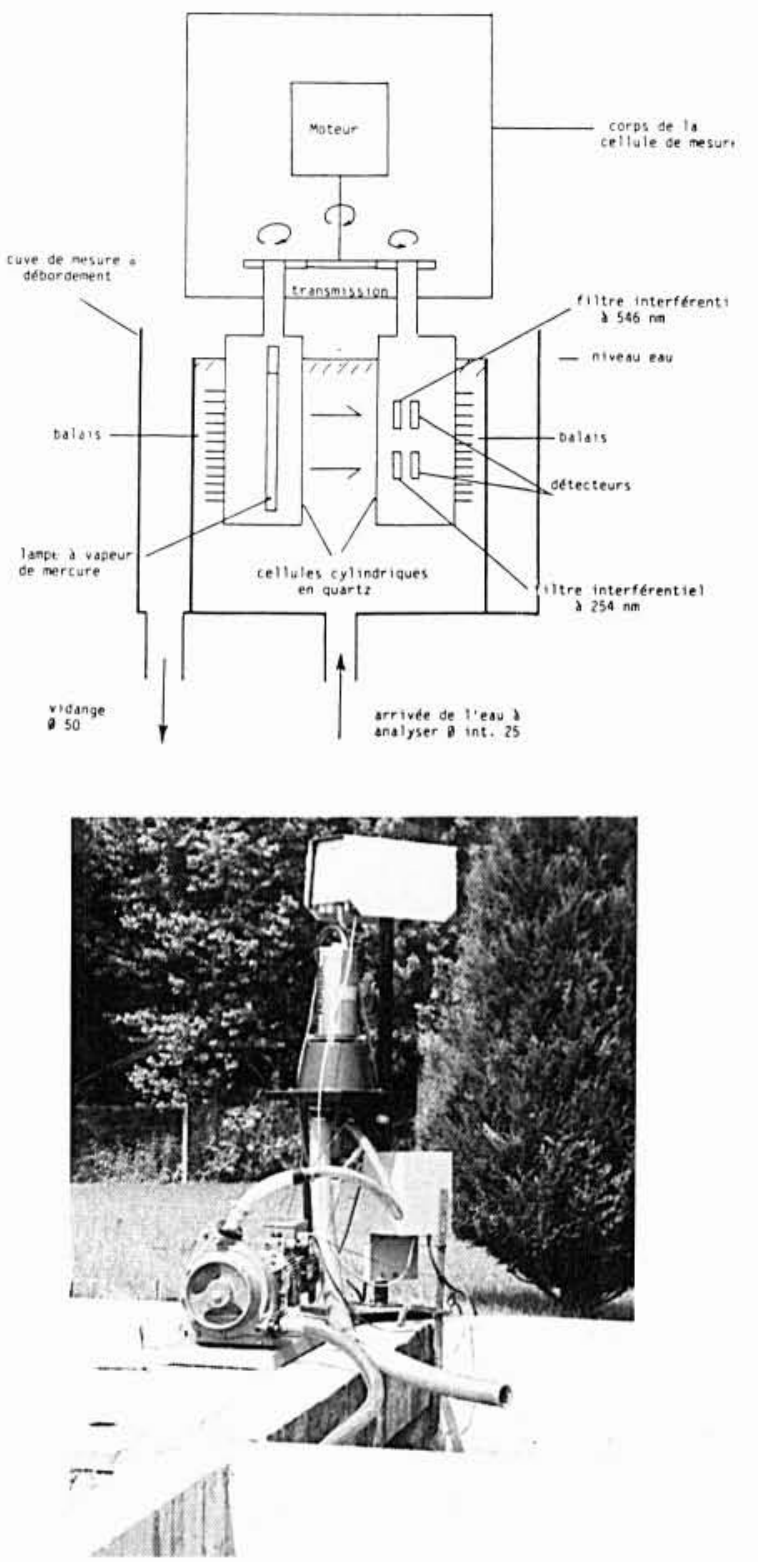

1. Appareil Horiba OPSA 100: Cellule de mesure.

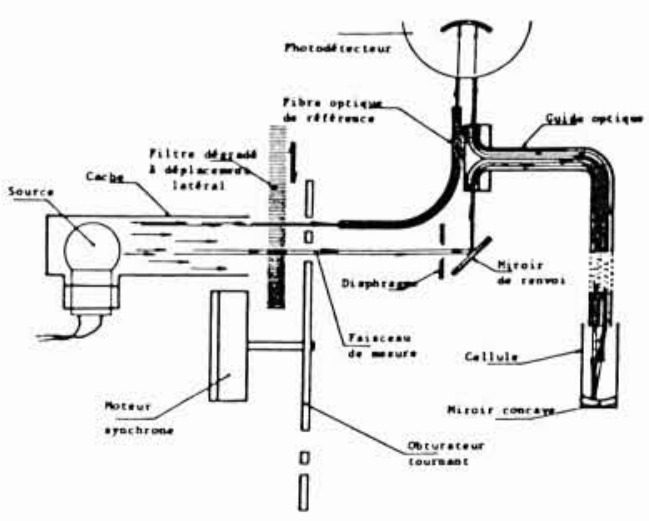

2. Photométre à fibre optique : Schéma de principe. 


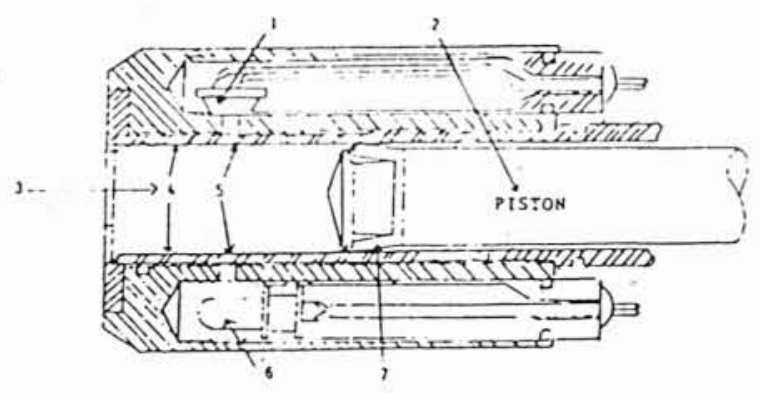

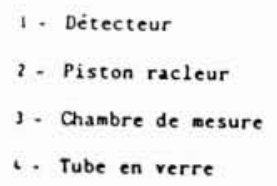

3. Turbidimètre " CLAM" à piston racleur: Tête de mesure.

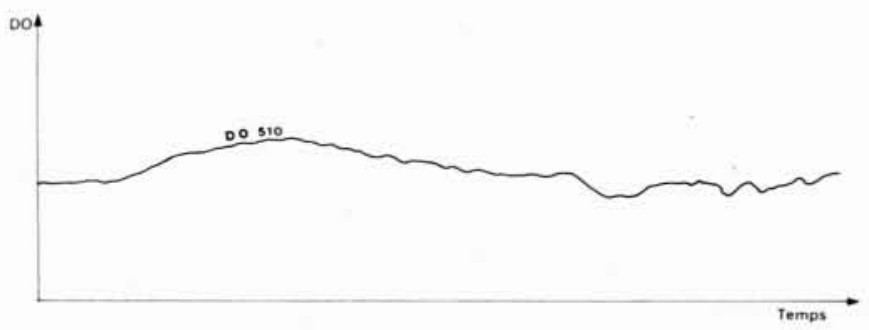

4. Mise en évidence de la pollution particulaire lors d'un épisode pluvieux.

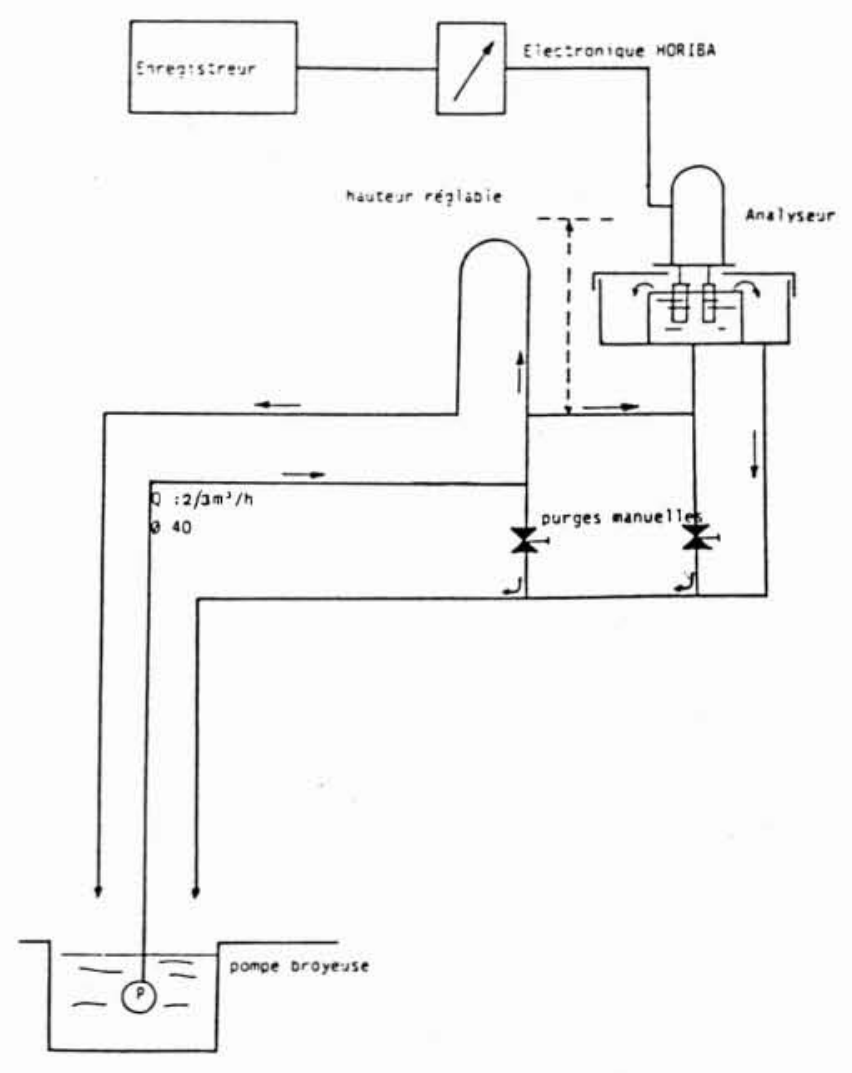

5. Appareil Horiba OPSA 100: Schéma hydraulique pour eau d'égout.

\subsection{Le spectrophotomètre Metrohm}

Ce spectrophotomètre à double faisceau dont la longueur d'onde d'émission peut varier entre 420 et $700 \mathrm{~nm}$ (visible) se distingue par sa rusticité et sa simplicité mais surtout par l'utilisation d'une fibre optique. La cuve de mesure classique est remplacée par un miroir qui renvoie la lumière incidente vers un guide optique. Les fibres optiques aller et retour, de section semi-circulaire, sont accolées et réunies en un guide unique qui aboutit à la cellule de mesure (fig. 2). Celle-ci est constituée d'un étrier en acier inoxydable supportant un miroir concave. Nous avons donc une cellule de mesure ouverte dont le chemin optique est de $20 \mathrm{~mm}$. L'appareil permet des mesures d'absorbance ou de transmission. Parmi les inconvénients de l'appareil, il convient de signaler que la fibre optique est trop courte, l'absence de dispositif d'intégration du signal et de zéro automatique.

\subsection{Description et caractéristiques du turbidimètre Monitek CLAM 52}

Le turbidimètre Moniiek CLAM est composé d'un ensem- ble capteur plus transmetteur. Le capteur est utilisé en immersion partielle dans un chenal ou un réseau, le transmetteur contient l'ensemble des circuits électroniques. Il est connecté au capteur par un câble multiconducteurs. L'originalité de ce matériel de mesure en continu de la turbidité est de s'affranchir d'une pompe de prélèvement, source de colmatage, et de fonctionner ainsi sur de longues périodes sans nettoyage manuel, ni maintenance.

La figure 3 représente une coupe en long de la tête du capteur montrant la source lumineuse, la cellule photorésistante, la chambre de mesure constituée d'un tube de verre pyrex. Le piston muni d'un joint râcleur vient nettoyer l'optique.

Le mouvement alternatif du piston permet le prélèvement de l'échantillon dans la chambre de mesure, puis le refoulement de l'effluent et le nettoyage de la chambre. Le cycle dure 15 secondes. D'après le constructeur, l'échelle de mesure s'étend entre 0,2 et $30 \mathrm{~g} / \mathrm{l}$. 


\section{Applications sur le terrain}

\subsection{Sur les eaux pluviales}

L'appareil Metrohm est installé à demeure sur le terrain depuis juillet 1985 pour effectuer des mesures sur un collecteur pluvial appelé " La Vieille Mer ». L'appareillage installé en mémoire est piloté par un microprocesseur industriel. La fibre plonge dans un bac alimenté par une pompe primaire protégée par un champ de grille. Nous n'avons rencontré aucun problème de fiabilité du matériel lors de l'année de mesure. Par contre l'encrassement de la cellule de mesure apparaît dès le deuxième épisode pluvieux et devient de plus en plus gênant dans le temps. Un cycle de lavage automatique après chaque épisode pluvieux est en cours d'installation.

\subsection{Sur les eaux usées}

\subsubsection{Essais de l'appareil Monitek}

Des essais ont été menés sur diverses stations recevant des effluents urbains et industriels (Limay, Porcheville, Nantes, Saint-Jean de Monts, Quiberon). Les coefficients de corrélation obtenus pour les relations entre la DCO et la turbidité Monitek ou entre les MES et cette turbidité sont tous supérieurs à 0,92 , excepté à Saint-Jean-de-Monts où le coefficient de corrélation entre DCO et turbidité n'est que de 0,88 . Du sulfure ferreux $(350 \mathrm{mg} / \mathrm{l})$ était injecté sur le réseau pour lutter contre les odeurs. Les sulfures coloraient en noir l'effluent en augmentant la turbidité mais sans nuire à la qualité de la relation obtenue. Un appareil Monitek sert depuis 3 ans à optimiser le traitement d'une station d'épuration physico-chimique en asservissant les ajouts de réactif à la charge des eaux traitée et à l'objectif de traitement recherché. Il n'a posé aucun problème d'encrassement.

\subsubsection{Essais de l'appareil Horiba OPSA 100}

Conçu pour fonctionner sur les eaux moyennement chargées telles que les eaux de rivière ou les eaux prétraitées de station d'épuration, l'appareil nécessite une adaptation pour son utilisation en eaux usées où les conditions de fonctionnement sont particulièrement défavorables.

Cette adaptation est fondamentale et a essentiellement porté sur la mise au point d'un circuit hydraulique d'alimentation fiable et robuste dont le coût reste raisonnable. La cuve à débordement a été surdimentionnée et le boitier électronique a été protégé de la corrosion par installation dans un coffret étanche. La Compagnie générale des eaux a testé le dispositif dans les réseaux de la région parisienne. Moyennant ces dispositions, l'appareil a permis de suivre avec une bonne fiabilité les variations de la charge polluante dans les effluents étudiés.

Par ailleurs l'analyseur a été mis en place sur différents sites de stations d'épuration.

L'alimentation en eaux décantées est plus aisée car les risques de bouchage du circuit hydraulique sont minimisés. De bonnes corrélations avec la DCO et la DBO5 ont pu être établies [9].

Ainsi pour un site donné, les équations suivantes ont été obtenues :
- pour la DCO : DCO = 213 (DO 254) + 1,4 (n = 38) La précision est de $13,6 \%$ (coefficient de variation évalué par le rapport de l'erreur standard sur la DCO moyenne); - pour la DBO5 : DBO5 = $165($ DO 254) $-73 \quad(n=48)$ La précision est de $9,6 \%$.

L'intégration de la mesure de l'absorption dans le visible n'améliore pas la précision de façon significative.

Les pentes diffèrent suivant les sites et il est nécessaire d'étalonner l'appareil en fonction de chaque type d'effluent.

L'appareil trouve une application intéressante pour l'aide à la conduite des traitements dans les stations d'épuration.

Ainsi la société OTV a développé, à partir de l'appareil OPSA 100, un procédé breveté d'Ecofloc qui permet l'asservissement des taux de réactifs à la charge polluante pour le traitement de floculation-coagulation dans les stations d'épuration physico-chimique. Une publication est actuellement en cours.

Le Laboratoire régional de l'Ouest Parisien à réalisé des mesures en station d'épuration à l'aide de l'appareil Horiba [10]. Les mesures ont été réalisées sur deux sites, en entrée et en sortie de station, pour montrer l'intérêt que présente la mesure continue de la qualité dans des cas particuliers tels que des apports industriels épisodiques ou un fonctionnement en limite de capacité :

La station $A$, de type boues activités moyenne charge avec décantation primaire, était alimentée par des eaux usées urbaines avec en outre les apports non permanents d'une distillerie liquorerie. (déstockages journaliers, pendant une période variable de quelques heures). L'effluent épuré dépassait souvent les normes.

La station $B$, de type chenal était alimentée par des eaux usées urbaines avec des apports d'une industrie de traitement de viande. On notait des fuites de boues épisodiques.

\subsubsection{Essais sur les eaux brutes}

Après décantation primaire (station $\mathrm{A}$ ), on a obtenu pour l'ensemble des 106 échantillons étudiés pendant deux semaines une corrélation de type :

$$
\text { DCO }=82,84=294(\text { DO254 }) \quad R^{2}=0,9084
$$

(la gamme de variation de la DCO est : 286 à $1540 \mathrm{mg} / \mathrm{l}$ )

Elle permet d'avoir une idée précise de la pollution organique arrivant à la station puisque l'écart entre DCO "AFNOR " et DCO "Ultra-violet " est inférieure à \pm $20 \%$ pour $90 \%$ des échantillons.

Par contre les corrélations entre les MES et la DO 546 sont de qualité très variables et parfois franchement médiocres.

D'autre part l'examen des enregistrements des mesures continues d'absorbance permet de mettre en évidence le caractère particulier du rejet.

L'enregistrement présenté en figure 6 (page suivante) montre bien que l'arrivée du rejet de la distillerie se traduit par une augmentation très nette de l'absorbance dans l'ultra-violet mais reste sans effet sur la turbidité.

Il s'avère donc impossible d'utiliser les bonnes corrélations DCO-DO 546 observées habituellement sur des eaux usées domestiques normales et régulières. 


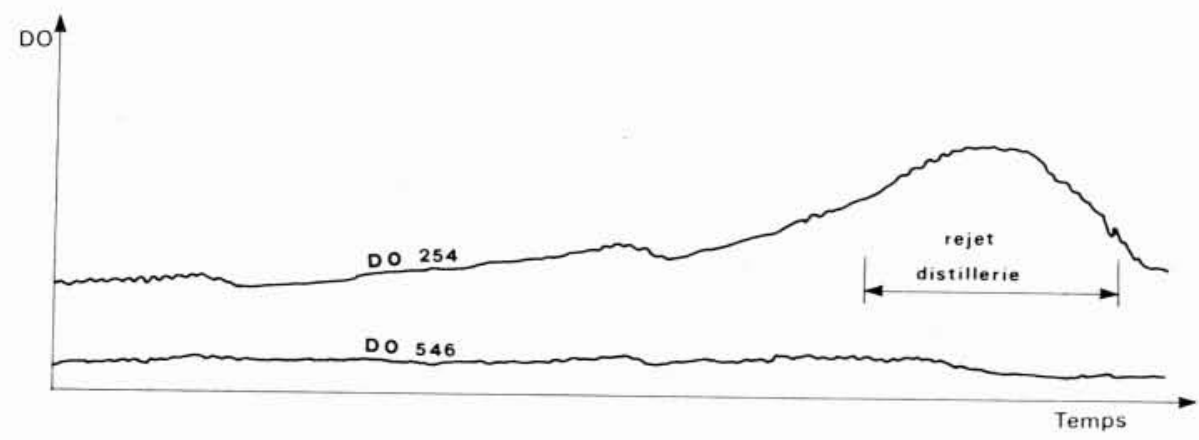

6. Mise en évidence d'une pollution organique soluble grâce à la mesure dans l'ultra violet.

\subsubsection{Essais sur les eaux épurées (stations $\mathrm{A}$ et $\mathrm{B}$ )}

Les corrélations linéaires DCO $\rightarrow$ DO 254 sont dans tous les cas d'excellente qualité. Il est donc possible de contrôler en continu avec un bon niveau de précision la conformité du rejet vis-à-vis des normes.

En ce qui concerne l'estimation des MES par mesures à $546 \mathrm{~nm}$, pour la station $\mathrm{A}$ (faibles valeurs, le plus souvent inférieures aux $30 \mathrm{mg} / 1$ réglementaires), les corrélations sont moins satisfaisantes; par contre pour la station B (où les valeurs élevées s'expliquent par des fuites de boues épisodiques) la très bonne corrélation obtenue permet d'envisager un bilan boues précis sur l'installation.

En conclusion, on retiendra globalement que sur les stations à problèmes - les plus intéressantes à suivre en continu - la mesure dans l'ultra-violet apporte un "plus " significatif par rapport à une mesure effectuée uniquement dans le visible.

\section{STATION A}

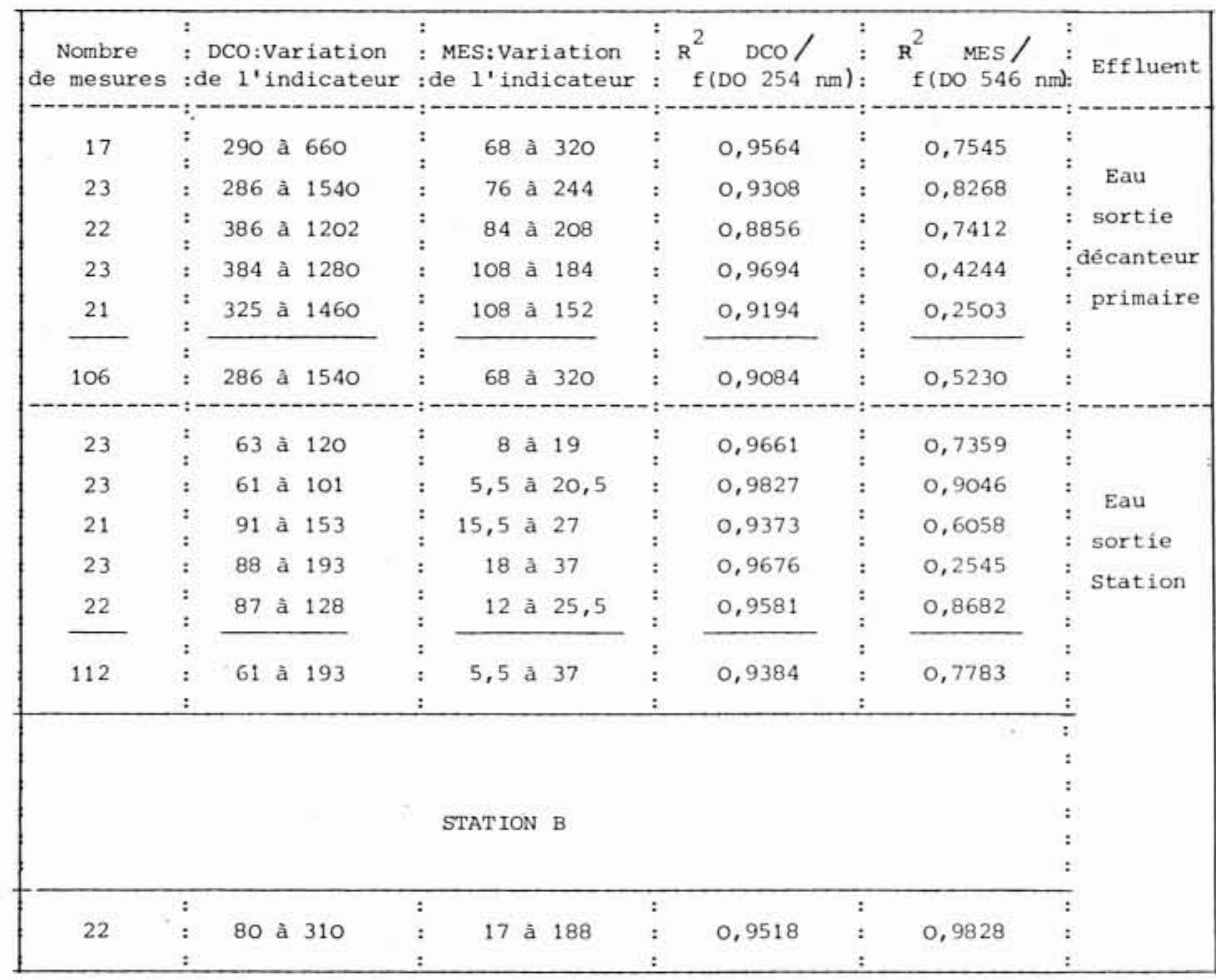




\section{Conclusion}

Il existe des appareils capables de donner une information corrélable avec la charge de l'eau. L'installation de ces matériels en réseau d'assainissement pour la mesure de qualité reste délicate alors que la mesure en station d'épuration sur eaux brutes ou sur eaux traitées (aux divers stades du traitement) est opérationnelle. Des constructeurs introduisent sur le marché de nouveaux appareils (ultrasons [11] des laboratoires de recherche travaillent également dans ce sens (électrode plus ultrasons). Cet exposé montre qu'au niveau national, des organismes privés ou publics travaillent à la mise au point d'un capteur de mesure continue et instantanée de la qualité de l'eau. Le développement de ce type de mesure dépendra du résultat de ces travaux bien sur, de la capacité des constructeurs à développer du matériel performant, fiable, facile à installer et bon marché, mais aussi de l'intérêt que les décideurs financiers voudront bien lui apporter (primes d'épuration en fonction des rendements des ouvrages, qualité des effluents industriels, gestion des réseaux).

\section{Bibliographie}

[1] Marchandise P., Legendre J.P., 1980. - Physico-chemical Treatment of waste water - Control or raegent additions by pollutional load to be treated. Progress in water technology, vpl. 12 , p. $359-369$

[2] Marchandise P., Legendre J.P., Delacroix D., Rivoal J.L., 1984. - Management of urban waste water physico-chemical treatment stations : servocontrol of reagent additions at the Quiberon sewage treatment plant. Water Science and Technology, vol. 17, p. 1103-1112.

[3] M. Mrkva, 1983. - Evaluation of correlation between absorbance at $254 \mathrm{~nm}$ and COD of river waters. Water Research 17, p. 231-235

[4] R.A. Dobis, R.H. Wise, R.B. DEAn, 1972. - The use of ultraviolet absorbance for monitoring the total organic carbon content of water and wastewater. Water Research, vol. 6. p. $1173-1180$

[5] Marchandise P., Legendre J.P., LAFOnT R., 1978. Méthode de mesure en continu de la pollution des eaux usées. La Technique de l'eau et de l'assainissement, $\mathrm{n}^{\circ} 383$, p. 11-18.

[6] Cathelain M., RobBe D., 1980. - Étude de la mesure de turbidité, test de turbidimètres. La Tribune du Cébédeau, $\mathrm{n}^{\circ}$ 438,33 , p. $251-268$

[7] BeN AIM R., MARTY J.L., COMA J., PARRAIN G., 1980 Utilisation de l'absorption ultra-violet à $254 \mathrm{~nm}$ pour la mesure en continu de la pollution organique dissoute. Analyse critique de l'analyseur $O P M$ - Tinsley.

[8] Grange D., Pescheux F., 1986. - Mesure en contimu de lo pollution organique et des matières en suspension par spectrophotomètre d'absorption dans l'ultraviolet. Etude Plan Urbain, Doc. LROP.

[9] F. Bourdon, J.M. Jestin, P. Ravarini, 1986. Utilisation de l'absorption ultraviolet pour l'estimation de la DCO et DBO5 dans les eaux.

[10] Grange D., Pescheux F. (à paraître). Utilisation de la spectrophotométrie d'absorption dans l'ultraviolet et le visible pour le contróle en continu du fonctionnement des stations d'épurations. Doc LROP-FAER 1-51-03-5.

[11] Defebre A., Pouliguen J., Marchandise P., 1985. Ultrasonic turbidimetry. Instrumentation and control of water and waste water tratment and transport systems. Edited by $R A R$ Drake, p. 543-546. 